\title{
Salt induced asymmetry in membrane simulations by partial restriction of ionic motion
}

\author{
Fernando E. Herrera ${ }^{1}$ and Sergio Pantano ${ }^{1,2, a)}$ \\ ${ }^{1}$ Institut Pasteur of Montevideo, Calle Mataojo 2020. CP 11400 Montevideo, Uruguay \\ and Consejo Nacional de Investigaciones Científicas y Técnicas (CONICET), Argentina \\ ${ }^{2}$ IMASL, CONICET, National University of San Luis, San Luis, Ejercito de los Andes 950, CP 4700, \\ San Luis, Argentina
}

(Received 26 September 2008; accepted 20 April 2009; published online 21 May 2009)

\begin{abstract}
The specific ionic composition differs considerably at both sides of biological membranes and specific lipid/electrolyte interactions may be essential for their structure, stability and function. Hence, explicit consideration of the ionic asymmetry is important to achieve an accurate description of lipid bilayers. Molecular dynamics simulations have proven to be a reliable tool to study biomembranes at atomic detail. Nevertheless, the use of periodic boundary conditions allows ions to diffuse rapidly and reach both sides of the bilayer. Therefore, ad hoc simulation schemes have to be applied to take into account ionic asymmetry. In this work we present a simple implementation to overcome this problem. A more realistic description of the biomembranes can be achieved by partially restricting the ionic motion in the direction normal to the membrane within a region of the space near to only one of the leaflets. This creates two different situations: one leaflet is highly exposed to ions while the second one can be completely or partially depleted of them. Comparison between this new method and control simulations performed using a previously proposed approach consisting of a double-membrane setup yielded an excellent agreement with a speed-up of nearly $60 \%$. The performance of the method with different ionic species is explored and remaining limitations are examined. (C) 2009 American Institute of Physics. [DOI: 10.1063/1.3132705]
\end{abstract}

\section{INTRODUCTION}

Biological membranes are systems of enormous complexity in terms of structural and dynamical properties because of their high heterogeneity. Their characterization still constitutes an important challenge to experimental techniques. However, the good agreement between experiments and results obtained by classical molecular dynamics (MD) studies on model membranes permitted applications to systems of a considerable degree of complexity. They include the explicit treatment of lipid-lipid, electrolyte-membrane, and membrane-protein interactions. ${ }^{1-5}$ Phospholipids' membranes are needed to separate different compartments within living cells containing different concentrations of molecules and ions. The presence of specific electrolytes may influence significantly the properties of biomembranes depending on their particular composition, local concentration of ions, their valence, etc. ${ }^{2,6-11}$ Since physiological conditions always imply electrolytes dissolved in aqueous solution, the problem of membrane/ions interactions is of fundamental interest. A careful consideration of the electrolytic asymmetry across the membrane must be taken into account to achieve a realistic description of a given membrane system by molecular simulations. ${ }^{2,10,12}$ However, the use of periodic boundary conditions (PBCs) in MD simulations results in the diffusion of ions across the box creating severe difficulties to repro-

\footnotetext{
${ }^{\text {a) }}$ Author to whom correspondence should be addressed. Telephone: +598 252209 10. Fax: $+598-252241$ 85. Electronic mail: spantano@pasteur.edu.uy.
}

duce the ionic asymmetry. Therefore, ad hoc approaches have to be implemented. The first attempt to explicitly address this topic consisted of the use of two parallel membrane planes in order to establish two separated aqueous compartments with different ionic concentrations [Fig. 1(a)]. It was originally proposed by Sachs et al. ${ }^{13}$ and successfully applied to a variety of systems, e.g., see Refs. 11, 12, and 14-19. This method effectively avoids the diffusion of ions and achieves a remarkable representation of the asymmetric conditions. However, the doubling of the system's size owing to the use of two membrane planes implies a considerable rise in the computational cost of the simulations. Alternatively, the so-called "slab geometry" scheme [Fig. 1(b)] has been proposed to model membrane-channel systems ${ }^{20}$ and recently applied to the study of the trans-membrane potential. ${ }^{21}$ In this scheme, single membrane systems are first stabilized at constant pressure, and then vacuum-water interfaces are introduced at both sides of the system by extending the computational box in the direction perpendicular to the membrane. After this stage, simulations are continued at constant volume. ${ }^{21}$ It has been shown that this method is efficient for generating trans-membrane currents avoiding a possible dipolar coupling between two consecutive PBC replicas. ${ }^{20}$ This method allows also for the physical separation between the two water compartments. Although, the use of constant volume instead of constant pressure conditions added to the limited diffusion of the solvent molecules in contact with the vacuum region may influence the dynamics of solvent and phospholipids. In the current study, we introduce an alternative strategy to simulate different ionic con- 


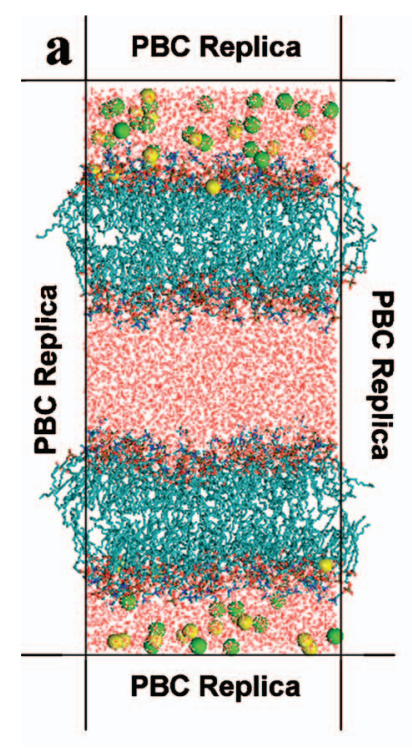

b

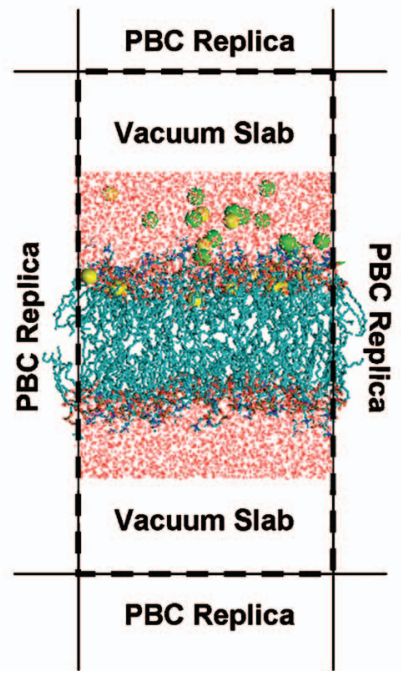

C

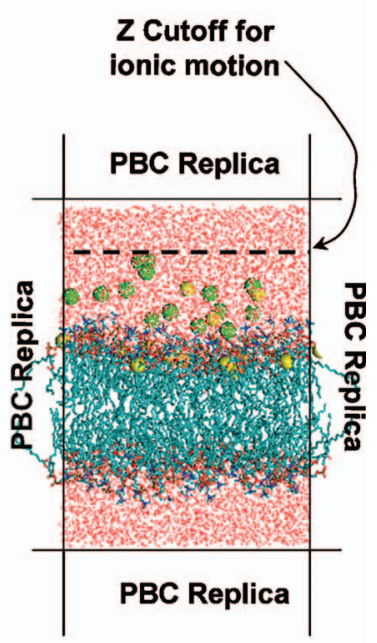

FIG. 1. (Color) Graphical presentation of the different approaches used to treat the asymmetric concentrations of ions by MD simulations. (a) Double membrane system scheme, (b) Slab geometry scheme, and (c) BRIM scheme. centrations across the membrane [Fig. 1(c)]. It consists of the use of a single phospholipids' bilayer with restricted ionic motion (BRIM) in the direction perpendicular to the membrane plane. In the BRIM scheme, PBCs are applied without the introduction of vacuum-water interfaces and simulations are always performed under NPT conditions. This allows a reduction in the system's size with respect to the double membrane systems ensuring correct diffusion of water molecules across the PBC images. The validation of the method was performed by comparison of the results of simulations on model membranes of dimyristoylphosphatidylcholine (DMPC) and $\mathrm{NaCl}$ salt using both BRIM and a double membrane system with unrestricted ions. The remarkable coherence of the results between both schemes points to the equivalence of the methods. Further we explored the performance of the method carrying out analogous simulations for systems containing divalent and nonadsorbing cations $\left(\mathrm{Ca}^{2+}\right.$ and $\mathrm{K}^{+}$, respectively). A comparison of the effect of different ions on the bilayer showed a good agreement with the data reported in the literature. ${ }^{2,11,22}$ Finally, remaining problems concerning the relaxation of both membrane leaflets exposed to different environments are discussed as well.

The aim of the present contribution is to introduce the BRIM method and compare its performance against a standard approach (i.e., the double membrane scheme). Therefore, only standard analysis and membrane compositions are presented in the current study. In the future, we would like to apply our method to more realistic and biologically relevant systems such as inhomogeneous membranes exposed to different ionic species.

\section{METHODS}

We designed a simple strategy to simulate the asymmetric concentration of ions across phospholipid bilayers using only a single membrane. As a reference for our method we used a standard procedure (double membrane) under homogeneous conditions. Our simulations were carried out on the basis of the setup presented by Gurtovenko. ${ }^{12}$ The following systems were simulated (see also Table I): (i) a single bilayer with pure water (no ions), (ii) one with $\mathrm{NaCl}$ salt freely diffusing at both sides of the bilayers, (iii) a double bilayer system with $\mathrm{NaCl}$ salt in one of the aqueous compartments, (iv) a single bilayer containing $\mathrm{NaCl}$ in which the ionic motion was partially restricted (BRIM scheme). The first three systems were used as controls and they were compared with system (iv). Moreover, in order to investigate the performance of the method with different ionic species (divalent and not adsorbed ions) other simulations were performed with: (v) a single bilayer containing $\mathrm{KCl}$ using the BRIM scheme and (vi) a single bilayer containing $\mathrm{CaCl}_{2}$ using the BRIM scheme. Finally, a single bilayer system containing $\mathrm{NaCl}$ using the BRIM scheme, in which the number of phospholipids on one leaflet was corrected according to the values of the area per lipid for the systems (i) and (ii) [system (vii)]. The last system was simulated to evaluate the effects of the variation in the area per lipid at both membrane sides upon exposition to ionic asymmetry (see Sec. IV).

In all the cases, the MD simulations were performed for $100 \mathrm{~ns}$, except for system (vi) (containing $\mathrm{CaCl}_{2}$ ), in which the simulation time was extended to $200 \mathrm{~ns}$ owing to the slower adsorption rate of the divalent cations. ${ }^{22}$

The membrane patches were composed of 64 DMPC phospholipid molecules on each leaflet (128 in total). The only exception was made for the system vii in which five phospholipids were removed from the monolayer not exposed to the electrolytes. The systems were solvated with

TABLE I. Summary concerning models investigated in the current study.

\begin{tabular}{lcccc}
\hline \hline System & Waters & DMPC & Ions & $\begin{array}{c}\text { Time } \\
\text { (ns) }\end{array}$ \\
\hline (i) & 5096 & 128 & $\ldots$ & 100 \\
(ii) & 5036 & 128 & $30 \mathrm{Na}^{+}-30 \mathrm{Cl}^{-}$ & 100 \\
(iii) & 10132 & 256 & $30 \mathrm{Na}^{+}-30 \mathrm{Cl}^{-}$ & 100 \\
(iv) & 6782 & 128 & $15 \mathrm{Na}^{+}-15 \mathrm{Cl}^{-}$ & 100 \\
(v) & 6782 & 128 & $7 \mathrm{Ca}^{2+}-14 \mathrm{Cl}^{-}$ & 200 \\
(vi) & 6782 & 128 & $15 \mathrm{~K}^{+}-15 \mathrm{Cl}^{-}$ & 100 \\
(vii) & 6782 & 123 & $15 \mathrm{Na}^{+}-15 \mathrm{Cl}^{-}$ & 100 \\
\hline \hline
\end{tabular}


TABLE II. Summary of the results obtained for the systems containing $\mathrm{Na}^{+}$ions.

\begin{tabular}{|c|c|c|c|c|c|c|c|c|c|}
\hline System & Water & DMPC & & Ions & $\begin{array}{l}\text { Time } \\
\text { (ns) }\end{array}$ & Area per lipid $\left(\AA^{2}\right)^{\mathrm{a}}$ & $\begin{array}{c}\text { Thickness } \\
(\AA)\end{array}$ & Cations adsorbed/leaflet ${ }^{\mathrm{b}}$ & $\begin{array}{c}\text { Diff. Coeff. } \\
\left(\mathrm{cm}^{2} / \mathrm{seg} 10^{-8}\right)^{\mathrm{c}}\end{array}$ \\
\hline (i) & 5096 & 128 & & $\ldots$ & 100 & $65.5 \pm 0.7$ & $33.7 \pm 0.4$ & 0 & $12.7 \pm 1.5$ \\
\hline (ii) & 5036 & 128 & 30 & $\mathrm{Na}^{+}-30 \mathrm{Cl}^{-}$ & 100 & $60.2 \pm 0.6$ & $35.8 \pm 0.5$ & 10.8 & $\begin{array}{c}9.0 \pm 1.4 \\
10.0 \pm 2.2\end{array}$ \\
\hline (iii) & 10132 & 256 & 30 & $\mathrm{Na}^{+}-30 \mathrm{Cl}^{-}$ & 100 & $63.5 \pm 0.5$ & $34.4 \pm 0.3$ & 10.8 & $\begin{array}{c}6.4 \pm 2.5 \\
17.7 \pm 1.3\end{array}$ \\
\hline (iv) & 6782 & 128 & 15 & $\mathrm{Na}^{+}-15 \mathrm{Cl}^{-}$ & 100 & $64.0 \pm 0.8$ & $34.0 \pm 0.5$ & 10.4 & $11.1 \pm 0.7$ \\
\hline (vii) & 6782 & 123 & 15 & $\mathrm{Na}^{+}-15 \mathrm{Cl}^{-}$ & 100 & $\begin{array}{l}67.0 \pm 0.9 \\
62.3 \pm 0.8\end{array}$ & $34.1 \pm 0.5$ & 10.6 & $\begin{array}{c}14.1 \pm 0.7 \\
8.1 \pm 0.7\end{array}$ \\
\hline
\end{tabular}

${ }^{\mathrm{a}}$ The area per lipid was calculated as the average $x$-y area along the trajectory divided by the number of lipids of each leaflet. For systems (i)-(iv), the area per lipid is the same for both leaflets. For system vii the two values correspond to the leaflet in contact with pure water (up) and electrolyte solution (down). ${ }^{\mathrm{b}}$ Calculated from the density profiles of Fig. 3(a) (see text).

${ }^{\mathrm{c}}$ The diffusion coefficient was averaged over the two leaflets in systems (i) and (ii). For systems (iii), (iv), and (vii) the values for the leaflet in contact with pure water (up) and salt solution (down) are shown.

SPC water molecules ${ }^{23}$ and the correspondent ionic pairs $\left(\mathrm{NaCl}, \mathrm{CaCl}_{2}, \mathrm{KCl}\right)$ were modeled using the default parameters from the GROMACs force field. ${ }^{24}$ The MD simulations were performed using the GROMACS package version 3.3.3. ${ }^{24,25}$ A direct cutoff for nonbonded interactions of $1 \mathrm{~nm}$ and particle mesh Ewald for long range electrostatics were applied. The Berendsen baths ${ }^{26}$ were used to couple the simulation boxes at a pressure of $1 \mathrm{~atm}$ and a temperature of $323 \mathrm{~K}$ to keep the bilayers in the liquid-crystalline phase. ${ }^{27}$ The lipid molecules and water/ions were coupled to separate Berendsen thermostats. All bond lengths of the lipid molecules were constrained using the LINCS algorithm, ${ }^{28}$ whereas the SETTLE algorithm ${ }^{29}$ was used for water molecules. The united atom force field of Berger et al. ${ }^{30}$ was applied for the phospholipids. The timestep was set to $2 \mathrm{fs}$.

A detailed composition of the systems is reported in Table I. The number of ions was chosen to roughly mimic the physiological conditions. In each specific system, randomly chosen water molecules were replaced by ions (see Table I). The simulations using the BRIM scheme had a separation between the average planes of both leaflets of nearly $4.5 \mathrm{~nm}$ (i.e., between two consecutive images within $\mathrm{PBCs}$ ). The ions of these systems were initially placed in one-half of the aqueous compartment, while the other half contained only pure water. During the MD simulation ions were allowed to move unconstrained in the directions parallel to the membrane ( $x$ and $y)$ and partially restricted in the direction normal to the membrane plane ( $z$ direction). This generates a kind of cutoff potential in the $z$ direction for the ions, with an increasing force restricting their movement to a region of the space near to one membrane leaflet. In order to implement this scheme within the GROMACS program, each ion was linked to a dummy atom (an atom that has no interactions with any other atom in the system) restricted to move only in the plane of the leaflet in contact with the ions. The functional form of the restraining potential was set to zero within a certain cutoff from the dummy atom. After that point, a spring constant was added up to a second cutoff, after which the harmonic restraint increased. Since the dummy atoms move only on the plane of one membrane leaflet, the chosen cutoff of $1.8 \mathrm{~nm}$ allowed the ions to move unconstrained within a perpendicular distance from the membrane below that value. The spring constant was added in the interval between 1.8 and $2 \mathrm{~nm}$, and force increased linearly after that point.

The cutoff distance was chosen to be $1.8 \mathrm{~nm}$ taking into account the fact that at least 25-30 water molecules per one phospholipid molecule are needed to maintain the correct phospholipids hydration; ${ }^{31}$ this corresponds to a solvation slab of nearly $1.2-1.5 \mathrm{~nm}$ on both sides of the membrane. Additionally, the radial distribution functions of water oxygen around ions of most of the common models for explicit water (e.g., TIP3P, SCP, SCP/E, etc.) converge to bulk values nearly after $0.8 \mathrm{~nm}$. Hence, a cutoff of $1.8 \mathrm{~nm}$ for the restraining potential guarantees that the ions-depleted leaflet will not feel any direct interaction from the ions restricted into the high concentration region.

To evaluate possible spurious effects of the restraining potential, the force constraint was varied from $10 \mathrm{KJ} / \mathrm{nm}^{2}$ to $10000 \mathrm{KJ} / \mathrm{nm}^{2}$ and four independent $50 \mathrm{~ns}$ long simulations were performed. The lowest value of $10 \mathrm{KJ} / \mathrm{nm}^{2}$ allowed the ions to reach the initially ion-depleted leaflet. Therefore a force constant of $100 \mathrm{KJ} / \mathrm{nm}^{2}$ was adopted as a minimum value to keep the asymmetric concentration of ions. Although larger values of the constant yielded essentially identical results, they increased the time needed by the systems to converge in terms of ion solvation and interaction with the membrane, likely due to the introduction of higher perturbations.

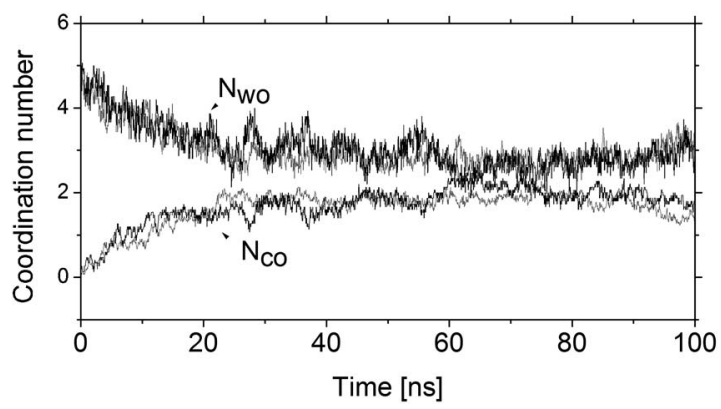

FIG. 2. Time evolution of coordination numbers of sodium ions with lipid carbonyl oxygen $\left(N_{\mathrm{CO}}\right)$ and water oxygen $\left(N_{\mathrm{WO}}\right)$ for the double membrane (black) and BRIM (gray) simulation schemes. 
Properties of ions adsorption and desolvation, density profiles, average area per lipid, electrostatic potential, and the orientation of the lipids headgroups and water molecules have been calculated over the last $20 \mathrm{~ns}$ of each simulation.

The average number of ions adsorbed on both leaflets was calculated from the density profiles as the area under the curves from the center of the membranes to the first minima in both distributions [Fig. 3(a) and Table II].

The diffusion coefficient of the phospholipids was calculated from the slope of the mean-square displacement of the center of mass (COM) of the lipids in the different monolayer planes along the trajectory. The slope was fitted between the $20 \%$ and $80 \%$ of the simulation time and the effects of the motion of the COM of the two monolayers relative to each other were removed for accuracy. The electrostatic potential across the simulation box [Fig. 3(d)] was calculated by integrating twice the total charge density of the systems along the $z$ axis. ${ }^{11,19,32,33}$ All the figures were done with VMD ${ }^{34}$ and QtiPlot (http://soft.proindependent.com/ qtiplot.html) programs.

\section{RESULTS}

\section{A. Asymmetric systems with $\mathrm{NaCl}$}

In the following paragraphs, a systematic comparison of the most characteristic parameters regarding membrane structure in presence of $\mathrm{NaCl}$ using the BRIM and double membrane schemes is presented. A good correspondence between the different simulation approaches highlights their equivalence.

\section{Ions adsorption/desolvation}

During the MD simulations some $\mathrm{Na}^{+}$ions leave the aqueous phase to get adsorbed into the membrane interacting with the polar heads of the phospholipids, while the $\mathrm{Cl}^{-}$ions remain free in solution. This adsorption/desolvation process is a measure of the equilibration of the system and it is considered to be the bottleneck for the simulations of a membrane under the influence of electrolytes. ${ }^{12,32}$ It can be directly monitored by following the time-course of the coordination number of $\mathrm{Na}^{+}$ions by water molecules and DMPC carbonyl oxygen atoms. As it is visible in Fig. 2, the coordination number of $\mathrm{Na}^{+}$ions with water oxygens $\left(N_{\mathrm{WO}}\right)$ decreased during the simulation time while the coordination number of $\mathrm{Na}^{+}$ions with carbonyl oxygen atoms $\left(N_{\mathrm{CO}}\right)$ increased, implying that some $\mathrm{Na}^{+}$ions get partitioned at the level of the carbonyl oxygen atoms. This process occurred with the same characteristic times, reaching nearly identical values in both simulation schemes.

\section{Density profiles}

The migration of $\mathrm{Na}^{+}$ions to the water/lipid interface is expected to modify the original distribution of the molecular species within the simulation box. To compare the final localization of the different species along the simulations and characterize the main features of the membrane structure, we measured the density profile of each of the components of the system along the $z$ direction. Figure 3(a) shows the den- sity profile for the double bilayer and BRIM simulations in the presence of $\mathrm{Na}^{+}$ions. Comparison of the density profiles highlights the good correspondence between both approaches, as it can be seen from the match of the density peaks for the DMPC, water, and ions. In particular, both setups describe equally well the deep penetration of sodium into the membrane down to the lipid carboxyl oxygen and the partition of chlorine into the aqueous face. The main difference between both methods resides in the obvious fact that the distribution of ions along the direction perpendicular to the membrane in the BRIM case is dumped by the restraining potential beyond the $z$ cutoff, while it rises with the distance from the bilayer reaching the bulk concentration in the middle of the aqueous compartment in the double membrane box [Fig. 3(a)]. Nonetheless, this effect does not modify the global association of the ions into the membrane. In fact, the average number of ions adsorbed on both leaflets is coincident (Table II).

\section{Area per lipid, membrane thickness, and diffusion coefficients}

The coordination of sodium by the carboxyl oxygen of the phospholipids exerts an ordering effect on the myristoil chains of DMPC since the lipid tails of two contiguous phospholipids are hold together due to the $\mathrm{Na}^{+}$mediated electrostatic interaction. ${ }^{35}$ The reduced mobility of the myristoil chains translates into a decrease in the average area per lipid in membranes exposed to sodium ions. This effect is clearly visible from Table II, where the higher area/lipid is observed for system (i) (no salt added), while the lowest value corresponds to system (ii) (salt added on both sides). ${ }^{33,36,37}$ Notably, both the double membrane and BRIM systems [(iii) and (iv), respectively] converged to an intermediate value. This indicates that the electrolyte-exposed bilayer tends to contract, shrinking the $x$ and $y$ sides of the simulation box. Therefore, the asymmetry introduced in both the BRIM and double membrane schemes drives the systems to a frustrated situation. The $x-y$ dimensions of the simulation box cannot reach equilibrium since the ions-exposed leaflet favors a contraction, while the pure-water leaflet favors an expansion. A possible solution to this problem is discussed in Sec. IV.

The reduction in the area/lipid translates into a progressive increase of the membrane thickness, measured as the average P-P distance between phosphorous atoms of the two monolayers (Table II). As expected, an opposite trend to that found for the area/lipid is retrieved for the thickness, with the lowest value corresponding to the system (i) (pure water), the highest for the system (ii) (ions on both sides), and intermediate values for the systems (iii) and (iv) (asymmetric conditions).

The electrolytes adsorbed onto the membrane influence also the lateral diffusion of the phospholipids in the bilayer plane. In agreement with previous data, ${ }^{32}$ we found that the addition of $\mathrm{Na}^{+}$ions reduce the mobility of the phospholipids in terms of their average diffusion coefficient (Table II). The obtained values are consistent with the experimental data in the literature ${ }^{38-40}$ although a considerable variability was observed among all the systems. This is probably a spurious effect of the relatively small size of the systems. ${ }^{41,42}$ Never- 

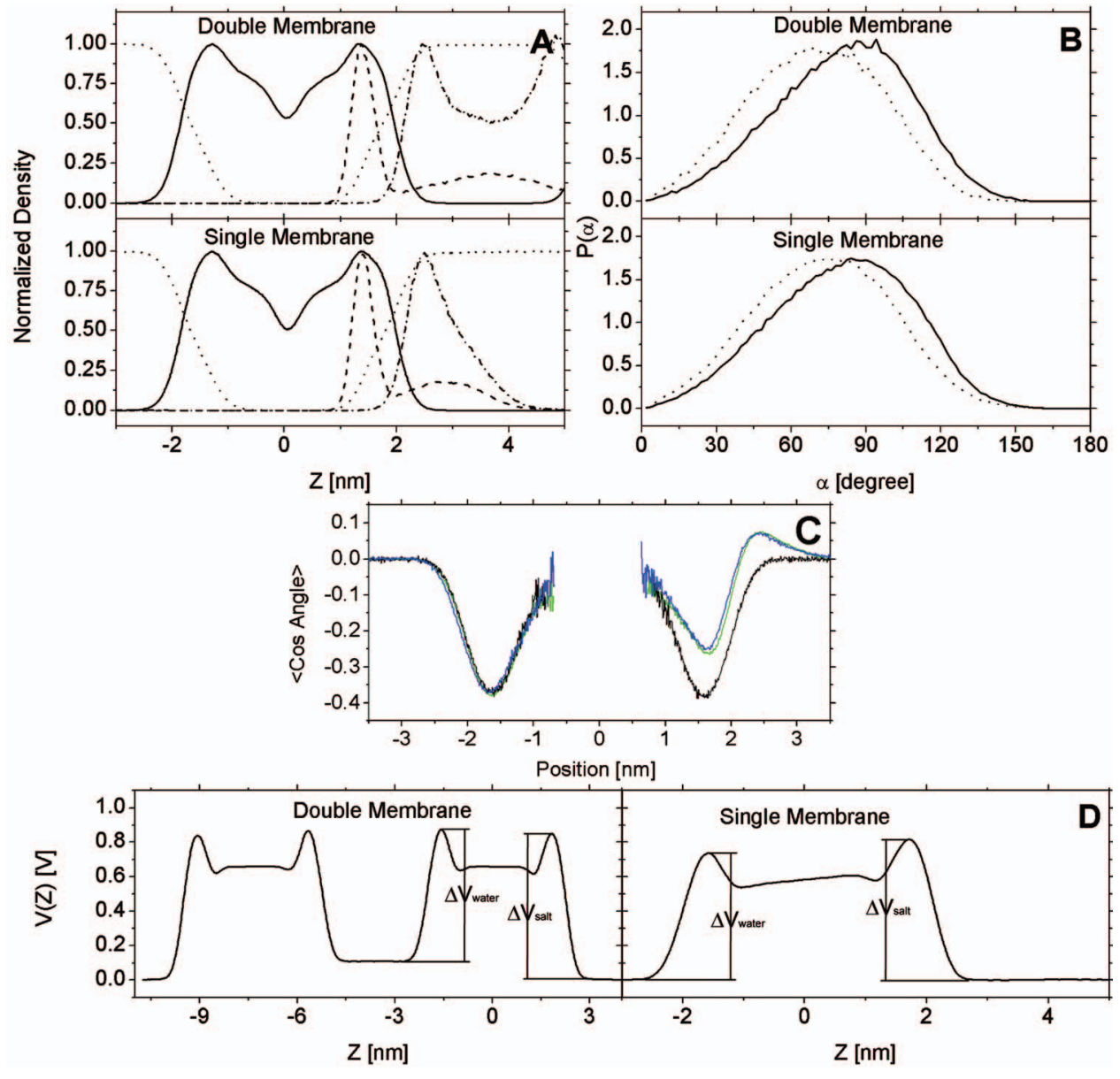

FIG. 3. (Color) Simulations with $\mathrm{NaCl}$ using the double membrane and the BRIM schemes: (a) Normalized number densities for the double membrane (top) and BRIM (bottom) systems, respectively. Number density profiles for DMPC (solid), $\mathrm{Na}^{+}$ions (dash), $\mathrm{Cl}^{-}$ ions (dash-dotted), and water molecules (dotted). (b) Distribution functions $\mathrm{P}(\alpha)$ of the angle between the $\mathrm{P}-\mathrm{N}$ vector of the lipid head groups and the outward bilayer normal for the double and single membrane systems. The results for the monolayers in contact with the water solution (solid line) and the monolayer in contact with salt solution (dashed line) are shown. $\mathrm{P}(\alpha)$ is given in arbitrary units. (c) Average orientation of water molecules with respect to the outward bilayer normal for the salt free system (black), the double membrane scheme (blue), and the BRIM scheme (green). (d) Electrostatic potential profiles $V(z)$ across the simulation box for the double membrane and the BRIM schemes. theless, both asymmetric schemes show a qualitative agreement as both retrieve a lower diffusion in the salt-exposed leaflets (Table II).

\section{Orientation of lipid head groups}

Incorporation of ions on the water/lipid interface not only modifies the structure and interphospholipid interactions, but also the membrane/solvent interactions as a consequence of the alteration of the average conformation of the phosphatidylcholine moieties. The zwitterionic head groups of DMPC posses a dipole moment along the $\mathrm{P}$ and $\mathrm{N}$ atoms. Thus the orientation of the head groups can be inferred from the average orientation of the P-N vectors with respect to the outward bilayer normal. Comparison between the orientations of the head groups located at different leaflets of the membrane provides information about the electrolyte driven reorganization of the membrane. Figure 3(b) shows the distribution function $\mathrm{P}(\alpha)$ for the angle between the $\mathrm{P}-\mathrm{N}$ vector and the outward bilayer normal for the double and single membrane (BRIM scheme) systems on both leaflets. As a result of the interaction with ions, a similar shift to lower values is observed for the salt-exposed leaflets in both asymmetric simulation schemes. ${ }^{10}$

\section{Orientation of water molecules}

The ionic adsorption and consequent reorganization of the polar head groups generate a perturbation that extends beyond the border of the phospholipids bilayer. A clear evi- dence of this fact is the ordering of the water molecules in the neighborhood of the bilayer. In the bulk, the water molecules do not have a preferential orientation. Hence, it is expected that $\langle\cos \phi\rangle=0$, where $\phi$ is the angle between the water dipole and a given orientation. However, if the orientation of the water molecules is calculated in the presence of a membrane and respect to the outward bilayer normal, negative values result for $\langle\cos \phi\rangle$ as a consequence of the hydrogen bonds between the oxygens of the phosphatidyl groups and the water hydrogens [see Fig. 3(c), left side]. As the distance from the membrane rise, the ordering effect vanishes and $\langle\cos \phi\rangle$ tends to zero. In the cases were the $\mathrm{Na}^{+}$is present, the orientation of water is significantly different. In these cases, the $\langle\cos \phi\rangle$ becomes positive after a certain distance as a result of the interactions with the adsorbed ions and the modified orientation of the phosphatidylcholine moieties [see Fig. 3(c), right side]. It is interesting to note that although $\mathrm{Na}^{+}$ions are deeply bound at the level of the carbonyl groups and the orientation of the P-N vectors is shifted to lower values, the perturbation in the water structure extends to more distant regions from the membrane plane in the presence of the salt [compare the left and right sides of Fig. 3(c)]. A nearly identical behavior is recovered for the BRIM and double membrane schemes.

\section{Electrostatic potential across the membrane}

A more stringent test for the performance of our method is the comparison of the electrostatic potential profiles across 

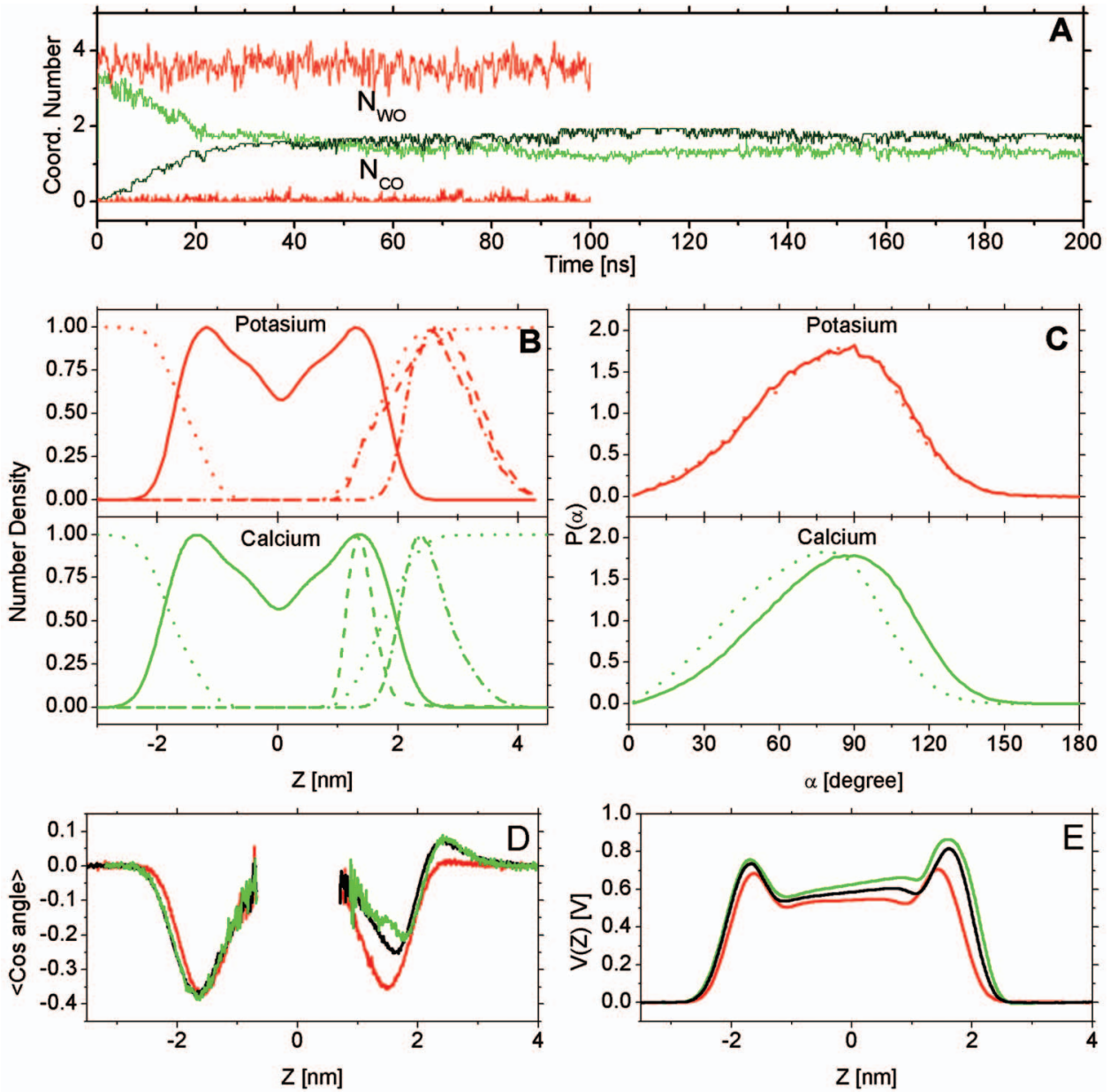

FIG. 4. (Color) Comparison of systems containing different electrolytes. Color codes are as follows: systems with $\mathrm{Na}^{+}$in black, systems with $\mathrm{K}^{+}$in red and systems with $\mathrm{Ca}^{2+}$ in green (for clarity, the $\mathrm{N}_{\mathrm{CO}}$ and $\mathrm{N}_{\mathrm{OW}}$ for $\mathrm{Ca}^{2+}$ are shown in light and dark green, respectively). (a) Time evolution of coordination numbers of the ions with lipid carbonyl oxygen $\left(N_{\mathrm{CO}}\right)$ and with water oxygen $\left(N_{\mathrm{WO}}\right)$. (b) Normalized number densities profiles for DMPC (solid), positive $\mathrm{K}^{+}$or $\mathrm{Ca}^{2+}$ ions (dash), negative $\mathrm{Cl}^{-}$ions (dashdotted), and water molecules (dotted). (c) Distribution functions $\mathrm{P}(\alpha)$ of the angle between the P-N vector of the lipids headgroups and the outward bilayer. The results for the monolayers in contact with the water solution (solid line) and the monolayer in contact with salt solution (dashed line) are shown. (d) Average orientation of water molecules with respect to the outward bilayer normal. (e) Electrostatic potential profiles $V(z)$ across the simulation box for the systems with $\mathrm{Na}^{+}$, $\mathrm{Ca}^{2+}$, and $\mathrm{K}^{+}$ions. the simulation box. This is particularly challenging, since there is a significant change in the symmetry of the double membrane case with respect to the BRIM scheme. In the first there are two separated aqueous compartments, while in the second only one aqueous phase exists, but with different ionic concentrations. Strictly speaking, the trans-membrane potential is defined as the difference between electrostatic potentials taken at two points located in the solution on the different sides of the membrane. In the case of one single membrane, the two solutions across the membrane are actually the same. However, the reorganization of water/lipid interface due to ionic adsorption generates a potential difference across the membrane, which is confined to the neighborhood of the membrane solution interface. A similar behavior has been found in simulations applying external potentials to membrane systems. ${ }^{43,44}$ In order to allow a direct comparison between the differences in electrostatic potential across the membrane in both simulation schemes, here it was defined as the difference between the electrostatic potential from the first peak within the membrane region to the aqueous phase at each side of the membrane [i.e., $\Delta V_{\text {salt }}-\Delta V_{\text {water }}$, where the subscripts salt and water refer to the membrane leaflets exposed and depleted of electrolytes, respectively, see Fig. 3(d)]. This assumption would suggest that the potential difference between the two peaks in the BRIM scheme could be compared to the proper transmembrane potential in double membrane systems. In fact, the values of the potential differences measured in the above defined way yield 80 and $90 \mathrm{mV}$ for the single and double membrane systems, respectively, in line with previous computational and experimental data. ${ }^{12,13,21}$

\section{B. Analysis of different ionic species}

Having assessed the capability of the BRIM scheme to reproduce the structural and dynamical features of membranes exposed to asymmetric ionic conditions for the case of adsorbing ions, we move our attention to electrolytes with different physicochemical characteristics.

First we concentrate on electrolyte species that do not interact with the membrane. To this aim we set up a simulation system containing $\mathrm{KCl}$, which is known to not attach to the bilayer due to its higher ionic radius. ${ }^{2,11}$ Indeed, $\mathrm{K}^{+}$ions remained confined to the aqueous phase as inferred from the water coordination number that oscillates around a constant value for the whole simulated time [Fig. 4(a)]. Only small peaks were found for the carbonyl oxygen coordination, which can be ascribed to short-lived contacts of the $\mathrm{K}^{+}$ions with the lipids due to Brownian motion in the aqueous phase. For this system, the density profile was very similar to that of the results reported for double membrane systems with POPC and POPE in contact to $\mathrm{KCl}$ salts $^{2}$ and for DPPC double membrane systems loaded with $\mathrm{Na}^{+}$and $\mathrm{K}^{+}$in each of the aqueous compartments, respectively. ${ }^{11}$ Briefly, the $\mathrm{K}^{+}$ ions showed no stiff interactions with the phospholipids bilayer remaining in the water phase [Fig. 4(b)]. In agreement 


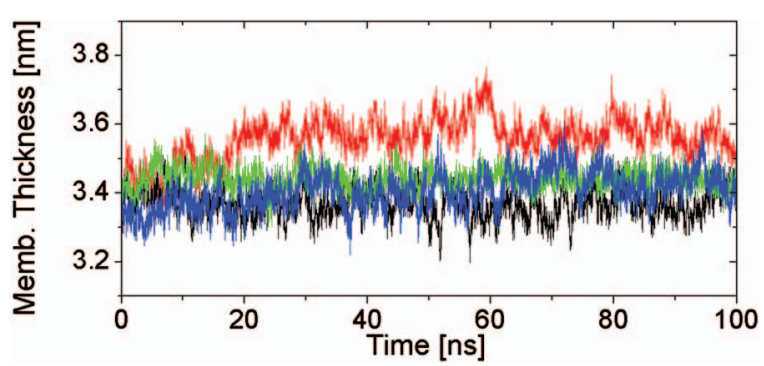

FIG. 5. (Color) Time evolution of the membrane thickness for $\mathrm{Na}^{+}$systems Color codes are as follows: salt free (black), salt (red), double membrane scheme (green), and BRIM scheme (blue).

with previous simulations, ${ }^{2,11}$ the distribution of $\mathrm{Cl}^{-}$ions is broader in the $\mathrm{KCl}$ case, as compared with the $\mathrm{NaCl}$ and $\mathrm{CaCl}_{2}$ cases [Figs. 3(a) and 4(b), respectively]. This may be a result of the more delocalized charge of cations in the solution, in contrast with the more positive potential created on the membrane plane by the adsorbed ions. As pointed out previously, in this case the ionic distributions $\left(\mathrm{K}^{+}\right.$and $\left.\mathrm{Cl}^{-}\right)$ were also dumped at the cutoff distance from the membrane. However, we were not able to find any associated spurious effects related to the spatial confinement of the ionic species. As a consequence of the lack of $\mathrm{K}^{+} /$membrane interaction, no shift is observed in the average orientation of the P-N vectors [Fig. 4(c)], nor differences in the water reorientation [compare the salt free trace in Fig. 3(b) with the potassium trace in Fig. 4(c)].

To enlarge the spectrum of possible interactions we simulated a system containing $\mathrm{CaCl}_{2}$. Divalent calcium cations have been reported to interact with the membrane in a similar fashion as that observed for $\mathrm{Na}^{+}$but longer stabilization times are needed. ${ }^{22}$ In agreement with this report, the $\mathrm{Ca}^{2+}$ adsorption took nearly $100 \mathrm{~ns}$ [Fig. 4(a)] while nearly $30 \mathrm{~ns}$ where needed for $\mathrm{Na}^{+}$(Fig. 2). After stabilization $\mathrm{Ca}^{2+}$ behaves in a very similar way as $\mathrm{Na}^{+}$. They remain bound to the membrane at the level of the carbonyl moieties leading to a similar density profile [compare Fig. 4(b) with Fig. 3(a)]. In line with the behavior of the $\mathrm{Na}^{+}$system, the divalent charge of the adsorbed $\mathrm{Ca}^{2+}$ induces a shift in the angular distribution of the P-N vectors toward smaller values [Fig. 4(c)]. A slightly more pronounced alteration at the membrane surface due to the divalent charge is suggested from the lower perturbation in the water orientation in the close neighborhood of the membrane [Fig. 4(d)], where the presence of $\mathrm{Ca}^{2+}$ partially screens the effect of the phosphate moieties reducing the water organization.

It is interesting to compare the electrostatic potential profile generated by the three studied cations. The superposition of the curves obtained for the systems containing $\mathrm{K}^{+}$, $\mathrm{Na}^{+}$, and $\mathrm{Ca}^{2+}$ [Fig. 4(e)] shows a clear increase in the potential differences generated by the accumulation of ions bound at the carbonyls moieties of DMPC, which is even more pronounced in the case of the $\mathrm{Ca}^{2+}$ simulation. The values for $V_{\mathrm{tm}}$ went from zero for the salt free case $10 \mathrm{mV}$ for $\mathrm{K}^{+}, 80 \mathrm{mV}$ for $\mathrm{Na}^{+}$, and $105 \mathrm{mV}$ for $\mathrm{Ca}^{2+}$. The $70 \mathrm{mV}$ difference between $\mathrm{K}^{+}$and $\mathrm{Na}^{+}$systems is coincident with that found in a multilamelar system containing $\mathrm{Na}^{+}$and $\mathrm{K}^{+}$ in each separated compartment. ${ }^{11}$ Confirming that the origin of the potential differences is the strong $\mathrm{Na}^{+}$binding to the phosphatidylcholine moieties. ${ }^{11}$ This effect is even more marked upon calcium adsorption [Fig. 4(e)], in qualitative agreement with the prominent role of divalent cations on the polarization of biological membranes.

\section{DISCUSSION}

One of the most important features of membrane simulations is the time needed for the equilibration. In this context, the association of cations with the lipid's carbonyl oxygen is often considered the bottleneck of membrane simulations. ${ }^{22,32}$ This process is usually measured, along the MD trajectories, as the desolvation of the ions as it is shown in Fig. 2. In the present study, it took about $30 \mathrm{~ns}$ for the desolvation curves to reach a plateau for both the BRIM and double membrane approaches (Fig. 2). Similarly, about $30 \mathrm{~ns}$ were needed for the single membrane simulation with freely diffusing electrolytes to equilibrate [system (ii), data not

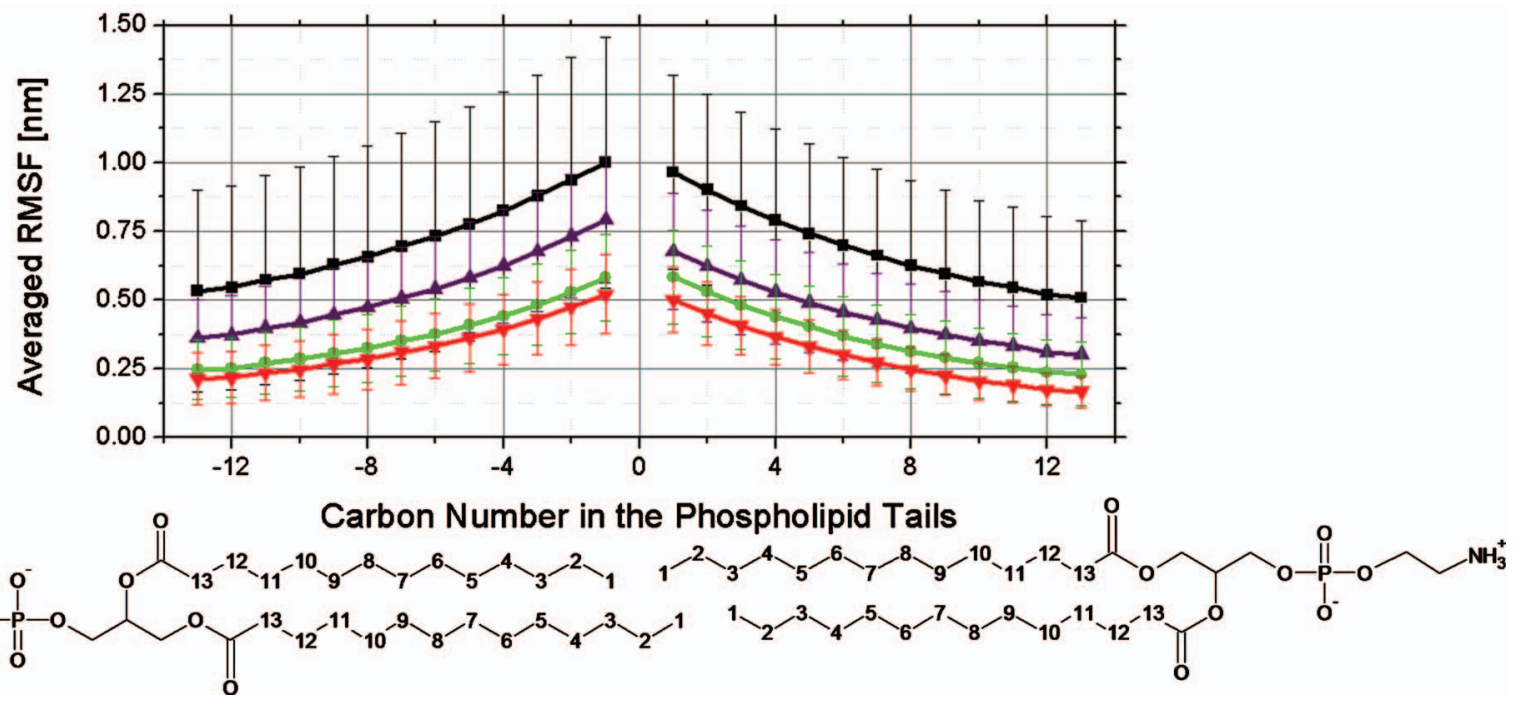

FIG. 6. (Color) Average RMSF along the trajectory for each of the carbon atom of the tail. The numeration of the atoms is shown in the scheme at the bottom. Color codes are as follows: salt free (black), salt (red), BRIM scheme (green), and corrected BRIM scheme (violet). 
shown]. Nevertheless, for the BRIM scheme and for the symmetric system (ii), the equilibration of the membrane thickness took longer times (Fig. 5). While $30 \mathrm{~ns}$ were needed for the double membrane to equilibrate, only after $\sim 50$ ns of simulation did the thickness in the simulated systems (ii) and (iv) reach the equilibrium (Fig. 5). This issue was further explored simulating a system containing divalent cations (i.e., $\mathrm{CaCl}_{2}$, Table I) for which much longer stabilization time in terms of ionic desolvation, nearly $100 \mathrm{~ns}$, has been reported. ${ }^{22}$ Accordingly, the ionic adsorption of $\mathrm{Ca}^{2+}$ ions stabilized around $100 \mathrm{~ns}$ [Fig. 4(a)]. This may suggest that somewhat longer stabilization times may be needed for the BRIM scheme as compared with the double membrane approach, although they are not relevant compared with state of art of simulation timescales used for the study of biological membranes. ${ }^{1,34-47}$ Furthermore, it points out that both the ionic desolvation/adsorption and the stabilization of the membrane thickness are needed to ensure the convergence of the system.

Another important problem related to simulations of asymmetric conditions in membranes is associated with the model construction: both leaflets of the membrane have the same area per lipid if the same number of phospholipids is used on both sides. It is well documented and also shown here that the area per lipid depends on the salt concentration (for systems containing $\mathrm{Na}^{+}$ions, Table II). Therefore, for a simulation box with asymmetric concentration of ions, neither the leaflet in contact with the salt nor the leaflet in contact with water would be totally equilibrated if both have the same number of phospholipids. This is because none of them can relax to its final area per lipid. Following the building procedure introduced by Gurtovenko and Vattulainen ${ }^{19}$ for heterogeneous phospholipids, Lee et al. ${ }^{11}$ proposed that a correction to the number of lipids in each leaflet could be applied to yield well equilibrated systems. This requires "preliminary simulations," which should be performed under symmetric conditions from which it is possible to get the corrected number of lipids for each leaflet. In order to further explore this issue and using the area per lipid from the control systems (i) and (ii), we constructed a new simulation box [system (vii)]. Considering the area per lipid of systems (i) and (ii) (65.5 and $60.2 \AA^{2}$, respectively), this led to a membrane with 64 lipids in the leaflet in contact with the salt and 59 lipids in the leaflet in contact with the water. For this new system, a $100 \mathrm{~ns}$ long MD simulation was carried out. The results obtained for the density number of the components of the system, the head groups, water orientation, and the electrostatic potential are very similar to those for the system (iv) (not shown). The results obtained for this new system are summarized in Table II. Although the area per lipid on both leaflets does not match exactly, the values of systems (i) and (ii) are closer than those obtained by the uncorrected simulations. The mismatch may be due to a finite size effect (strictly a noninteger number of phospholipids should had been removed). Alternatively, the mismatch may also originate from the interactions between the lipid tails, which have a different mobility (notice the difference in the diffusion coefficient, Table II). This suggests a sort of coupling between leaflets where the salt-exposed lipids tend to immobi- lize the water-exposed lipids, while the latter tend to agitate the former. Therefore, each monolayer under ionic asymmetry would behave differently one from each other, but also differently from the control systems. If this were the situation, it would never be possible to reach a perfectly equilibrated system since the impossibility to relax it is implicit in the application of PBCs and independent on the method used to simulate the ionic asymmetry. The hypothesis is supported by results obtained analyzing the root mean square fluctuation (RMSF) of the carbon atoms of the lipid tails. Comparison of the RMSFs between systems (i), (ii), (iv), and (vii) (Fig. 6) suggests that the lipids in the corrected system (vii) have a mobility which is in the middle between those of the salt-free (i) and symmetric salt-added (ii). However, it is worth noticing that the differences obtained in the area/lipid upon correction of the number of lipids is still in the order of the uncertainty of the state of art experimental techniques, ${ }^{48,49}$ making this issue difficult to tackle.

\section{CONCLUSIONS}

Despite the important success obtained by MD simulations in description of biomembranes and the exponential growth of computer power, finite-size artifacts are still a matter of concern (see discussion in Ref. 11). Therefore, new methods aimed to reduce the computational cost of simulations, bridging the in silico with the in vitro/in vivo relevant timescales are still needed. In this contribution, we presented a simple and timesaving scheme to model ionic asymmetry across phospholipids bilayers. The BRIM method introduced here nicely reproduces experimental data and results from analogous simulations performed using the standard double membrane scheme. Comparison of the performance of both strategies showed a speedup of nearly $60 \%$ due to the reduced number of atoms in the BRIM case, which represents a clear advantage. Hence, the method presented here provides a computationally cheap tool to accurately investigate a major determinant of biomembranes structure/dynamics, which is often neglected in molecular dynamic simulations.

\section{ACKNOWLEDGMENTS}

This work was supported by ANII-Agencia Nacional de Investigación e Innovación, Progama de Apoyo Sectorial a la Estrategia Nacional de Innovación-INNOVA URUGUAY (Agreement no. DCI-ALA/2007/19.040 between Uruguay and the European Commission).

\footnotetext{
${ }^{1}$ S. A. Pandit, E. Jakobsson, and H. L. Scott, Biophys. J. 87, 3312 (2004).

${ }^{2}$ A. A. Gurtovenko and I. Vattulainen, J. Phys. Chem. B 112, 1953 (2008).

${ }^{3}$ C. F. Lopez, S. O. Nielsen, B. Ensing, P. B. Moore, and M. L. Klein, Biophys. J. 88, 3083 (2005).

${ }^{4}$ D. E. Chandler, J. Hsin, C. B. Harrison, J. Gumbart, and K. Schulten, Biophys. J. 95, 2822 (2008).

${ }^{5}$ P. Sane, E. Salonen, E. Falck, J. Repakova, F. Tuomisto, J. M. Holopainen, and I. Vattulainen, J. Phys. Chem. B 113, 1810 (2009).

${ }^{6}$ R. J. Clarke and C. Lupfert, Biophys. J. 76, 2614 (1999).

${ }^{7}$ M. Rappolt, G. Pabst, H. Amenitsch, and P. Laggner, Colloids Surf., A 183-185, 171 (2001)

${ }^{8}$ H. Binder and O. Zschornig, Chem. Phys. Lipids 115, 39 (2002).

${ }^{9}$ A. A. Gurtovenko, M. Patra, M. Karttunen, and I. Vattulainen, Biophys. J. 86, 3461 (2004).

${ }^{10}$ A. A. Gurtovenko, M. Miettinen, M. Karttunen, and I. Vattulainen, J.
} 
Phys. Chem. B 109, 21126 (2005).

${ }^{11}$ S. J. Lee, Y. Song, and N. A. Baker, Biophys. J. 94, 3565 (2008).

${ }^{12}$ A. A. Gurtovenko, J. Chem. Phys. 122, 244902 (2005).

${ }^{13}$ J. N. Sachs, P. S. Crozier, and T. B. Woolf, J. Chem. Phys. 121, 10847 (2004).

${ }^{14}$ A. A. Gurtovenko and I. Vattulainen, J. Am. Chem. Soc. 127, 17570 (2005).

${ }_{15}^{15}$ A. A. Gurtovenko and I. Vattulainen, Biophys. J. 92, 1878 (2007).

${ }^{16}$ S. K. Kandasamy and R. G. Larson, J. Chem. Phys. 125, 074901 (2006).

${ }^{17}$ S. Leekumjorn and A. K. Sum, Biochim. Biophys. Acta 1758, 1751 (2006).

${ }^{18}$ P. T. Vernier, M. J. Ziegler, Y. Sun, M. A. Gundersen, and D. P. Tieleman, Phys. Biol. 3, 233 (2006).

${ }^{19}$ A. A. Gurtovenko and I. Vattulainen, J. Phys. Chem. B 112, 4629 (2008).

${ }^{20}$ D. Bostick and M. L. Berkowitz, Biophys. J. 85, 97 (2003).

${ }^{21}$ L. Delemotte, F. Dehez, W. Treptow, and M. Tarek, J. Phys. Chem. B 112, 5547 (2008).

${ }^{22}$ R. A. Bockmann and H. Grubmuller, Angew. Chem. Int. Ed. Engl. 43, 1021 (2004)

${ }^{23}$ H. J. Berendsen, J. R. Grigera, and T. P. Straatsma, J. Phys. Chem. 91, 6269 (1987)

${ }^{24}$ E. Lindhal, B. Hess, and D. van der Spoel, J. Mol. Model. 7, 306 (2001).

${ }^{25}$ D. van der Spoel, E. Lindahl, B. Hess, G. Groenhof, A. E. Mark, and H. J. Berendsen, J. Comput. Chem. 26, 1701 (2005).

${ }^{26}$ H. J. C. Berendsen, J. P. M. Postma, W. F. van Gunsterren, A. DiNola, and J. R. Haak, J. Chem. Phys. 81, 3684 (1984).

${ }^{27}$ G. Cevc and D. Marsh, Phospholipid Bilayers: Physical Principles and Models (Wiley, New York, 1987).

${ }^{28}$ B. Hess, H. Bekker, H. J. Berendsen, and J. Fraaije, J. Comput. Chem. 18, 1463 (1997)

${ }^{29}$ S. Miyamoto and P. A. Kollman, J. Comput. Chem. 13, 952 (1992).

${ }^{30}$ O. Berger, O. Edholm, and F. Jahnig, Biophys. J. 72, 2002 (1997).
${ }^{31}$ H. I. Petrache, S. Tristram-Nagle, and J. F. Nagle, Chem. Phys. Lipids 95, 83 (1998).

${ }^{32}$ R. A. Bockmann, A. Hac, T. Heimburg, and H. Grubmuller, Biophys. J. 85, 1647 (2003).

${ }^{33}$ S. A. Pandit, D. Bostick, and M. L. Berkowitz, Biophys. J. 84, 3743 (2003).

${ }^{34}$ W. Humphrey, A. Dalke, and K. Schulten, J. Mol. Graphics 14, 33 (1996).

${ }^{35}$ P. Mukhopadhyay, L. Monticelli, and D. P. Tieleman, Biophys. J. 86, 1601 (2004).

${ }^{36}$ J. F. Nagle, R. Zhang, S. Tristram-Nagle, W. Sun, H. I. Petrache, and R. M. Suter, Biophys. J. 70, 1419 (1996).

${ }^{37}$ S. C. Costigan, P. J. Booth, and R. H. Templer, Biochim. Biophys. Acta 1468, 41 (2000).

${ }^{38}$ W. L. Vaz, R. M. Clegg, and D. Hallmann, Biochemistry 24, 781 (1985).

${ }^{39}$ P. F. Almeida, W. L. Vaz, and T. E. Thompson, Biochemistry 31, 6739 (1992).

${ }^{40}$ A. Filippov, G. Oradd, and G. Lindblom, Biophys. J. 84, 3079 (2003).

${ }^{41}$ M. Patra, M. Karttunen, M. T. Hyvonen, E. Falck, P. Lindqvist, and I. Vattulainen, Biophys. J. 84, 3636 (2003).

${ }^{42}$ E. Falck, M. Patra, M. Karttunen, M. T. Hyvonen, I. Vattulainen, Biophys. J. 87, 1076 (2004).

${ }^{43}$ B. Roux, Biophys. J. 95, 4205 (2008).

${ }^{44}$ P. Bjelkmar, P. S. Niemela, I. Vattulainen, and E. Lindahl, PLOS Comput. Biol. 5, e1000289 (2009)

${ }^{45}$ P. S. Niemela, O. Ollila, M. T. Hyvonen, M. Karttunen, and I. Vattulainen, PLOS Comput. Biol. 3, e34 (2007).

${ }^{46}$ S. A. Pandit and H. L. Scott, Methods Mol. Biol. 398, 283 (2007).

${ }^{47}$ M. L. Klein and W. Shinoda, Science 321, 798 (2008).

${ }^{48}$ E. A. Disalvo, F. Lairion, F. Martini, E. Tymczyszyn, M. Frias, H. Almaleck, and G. J. Gordillo, Biochim. Biophys. Acta 1778, 2655 (2008).

${ }^{49}$ F. Lairion and E. A. Disalvo, J. Phys. Chem. B 113, 1607 (2009). 